\title{
FROUXIDÃO LIGAMENTAR E PÉ PLANO EM CRIANÇAS NORMAIS
}

\section{LIGAMENT LAXITY AND FLATFOOT IN NORMAL CHILDREN}

Guilherme Guadagnini Falotico', Maurício Takashi de Lima Uyeda', Renata Aparecida Leonel Romão', Alex Sandro Peres de Freitas ${ }^{2}$, Francesco Camara Blumetti ${ }^{3}$, Eiffel Tusuyoshi Dobashi ${ }^{4}$, José Antonio Pinto ${ }^{5}$

\section{RESUMO}

Objetivo: Avaliar a relação entre o pé plano flexível e a frouxidão ligamentar em crianças hígidas. Métodos: Foram examinadas 328 crianças entre três e 15 anos, de ambos os sexos, sem comprometimento do sistema musculoesquelético. Utilizamos os critérios de Beighton e Horan para determinação da frouxidão ligamentar e a presença do pé plano segundo a classificação de Valenti. Correlacionamos os dados coletados de acordo com o sexo, a idade, o grupo étnico e o lado dominante, e os resultados foram submetidos à análise estatística pelo teste do Qui-quadrado e teste exato de Fischer. Resultados: Observamos um percentual de $83,9 \%$ indivíduos portadores de frouxidão quando a idade menor que sete anos $\left(\mathrm{p}<0,001^{*}\right)$. Há associação estatística significante entre a presença de frouxidão e sexo $\left(\mathrm{p}=0,025^{*}\right)$, sendo que o feminino apresenta percentual maior de frouxidão $(51,02 \%)$. Observamos uma associação significante entre a frouxidão e o tipo de pé ( $\mathrm{p}$ $=0,003^{*}$ ), pois o grupo com pé plano apresentou um percentual maior de frouxidão $(54,96 \%)$. O pé plano também esteve associado à frouxidão considerando o sexo masculino $(\mathrm{p}=0,001 *)$, o que não foi observado no sexo feminino. Conclusão: As crianças com idade menor que sete anos, indivíduos do sexo feminino e os portadores de pé plano apresentam maior frequência de frouxidão ligamentar na população estudada. Não há associação entre a frouxidão, grupo étnico e lado dominante.

Descritores - Instabilidade Articular; Pé Chato; Criança

\section{ABSTRACT}

Objective: To study the correlation between flatfoot and joint laxity in healthy children. Methods: We evaluated 328 children with ages between 3 to 15 years, with no previous musculoskeletal complaints. We classified them by the presence of joint laxity according to the Beighton and Horan criteria, and by the presence of flatfoot according to the Valenti classification. The data obtained werecorrelated with gender, age, ethnic group, and dominant side. Fisher's exact test and chi-square test were applied to analyze theresults. Results: A percentage of $83.9 \%$ ofindividuals with joint laxity was observed in children younger than 7 years of age $(p<0.001 *)$. There is a significant association between joint laxity and gender $\left(p=0.025^{*}\right)$, as girlsexhibited a greater percentage of laxity (51.02\%). We observed a significant association between joint laxity and type of foot $(p=0.003 *)$, since the flatfooted group presented a higher percentage of laxity $(54.96 \%)$. Flatfoot was also associated to joint laxity when we considered only the males $(p=0.001 *)$, which was not observed in the females group. Conclusion: In the population studied, joint laxity was more frequently observed in children younger than 7 years of age, in females, and in individuals with flatfoot. There was no association between joint laxity and ethnic group or dominant side.

Keywords - Joint Instability; Flatfoot; Child

\section{INTRODUÇÃO}

A hipermobilidade articular tem relevante importância na prática diária do ortopedista; o interesse por esta condição iniciou-se no fim do século XIX, quando esta condição era considerada patológica ${ }^{(1,2)}$.
Na população normal, há grande variação das medidas da amplitude de movimento articular que, geralmente, é maior nos indivíduos do sexo feminino e também é influenciada pelas diferenças étnicas ${ }^{(3-6)}$, entre outros aspectos. A flexibilidade é um dos com-

1 - Médicos Residentes do Departamento de Ortopedia e Traumatologia da Universidade Federal de São Paulo.

2 - Médico Ortopedista pela Universidade Federal de São Paulo; Membro titular da Sociedade Brasileira de Ortopedia e Traumatologia.

3 - Médico Assistente da Disciplina de Ortopedia Pediátrica do Departamento de Ortopedia e Traumatologia da Universidade Federal de São Paulo

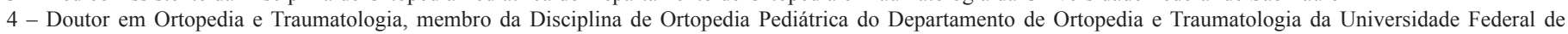
São Paulo.

5 - Professor Adjunto da Disciplina de Ortopedia Pediátrica do Departamento de Ortopedia e Traumatologia da Universidade Federal de São Paulo.

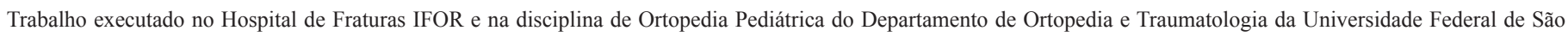
Paulo - Escola Paulista de Medicina.

Correspondência: Rua Borges Lagoa, 783 - 5ªndar - Vila Clementino - 04038-032 - São Paulo, SP. E-mail: ggfalotico@yahoo.com.br 
ponentes da aptidão física e pode ser definida como a amplitude fisiológica passiva máxima de um determinado movimento $\operatorname{articular}^{(7,8)}$, diminuindo conforme a progressão da idade ${ }^{(3)}$.

Diversos métodos de avaliação foram desenvolvidos para a análise clínica da flexibilidade articular. Entre eles, a sistemática descrita por Beighton e Horan $^{(9)}$ apresenta a maior aplicação na prática diária na área de saúde.

Entre as muitas afecções ortopédicas existentes, o pé plano (PP) é uma das que têm despertado um grande interesse pelos diversos autores da literatura ortopédica. É referido que tal condição está associada com a hipermobilidade articular e, portanto, com a frouxidão ligamentar, embora esta correlação não esteja totalmente esclarecida. Da mesma maneira, sua etiologia ainda permanece desconhecida ${ }^{(9)}$.

O termo pé plano (PP) é frequentemente usado para descrever uma variedade de situações associadas com as mais diversas condições patológicas ${ }^{(10)}$. Sua avaliação depende de uma análise minuciosa de diversos elementos, tais como: história clínica; exame físico, com especial atenção para a frouxidão articular, posturas angulares e rotacionais dos membros inferiores; avaliação dos calçados; posição da patela; mobilidade em extensão do hálux; alinhamento do hálux; encurtamento do tendão calcâneo; calosidades dos pés; podograma; documentação fotográfica ${ }^{(10-12)}$.

Como são escassos, os trabalhos que avaliam o binômio PP e frouxidão ligamentar em crianças normais, desenvolvemos este estudo, tendo como objetivo analisar esta relação, considerando-se também o sexo, a idade, o grupo étnico e o lado dominante.

\section{MATERIAL E MÉTODOS}

Inicialmente, este projeto de pesquisa foi submetido ao Comitê de Ética Médica em Pesquisa desta instituição e aprovado para realização.

Fazem parte deste material 328 crianças provenientes de uma instituição de ensino fundamental do Estado de São Paulo. Como critério de inclusão, as mesmas deveriam ser assintomáticas, ou seja, sem anormalidades do sistema musculoesquelético pregressas ou atuais. A idade variou de três a 15 anos, sendo divididas em dois grupos distintos e homogêneos de acordo com o sexo, sendo 145 (44,2\%) femininos e $183(55,8 \%)$ masculinos. Em relação ao grupo étnico, $113(34,5 \%)$ eram brancos e $215(65,5 \%)$ não brancos. Quanto ao lado,
$298(90,9 \%)$ apresentavam como dominante o direito e $30(9,1 \%)$, o esquerdo.

A frouxidão ligamentar foi estimada pelo exame clínico, usando medidas adimensionais da amplitude de movimento articular conforme preconizado por Beighton e Horan ${ }^{(9)}$. De acordo com este método, a mobilidade articular é determinada pelo somatório de números inteiros, que varia de 0 a 9 pontos. É dado um ponto para a capacidade de efetuar movimentos específicos dos membros superiores e inferiores, considerando-se o lado dominante e o não dominante e, por fim, a mobilidade do tronco. Os elementos avaliados são os seguintes (Figura 1):

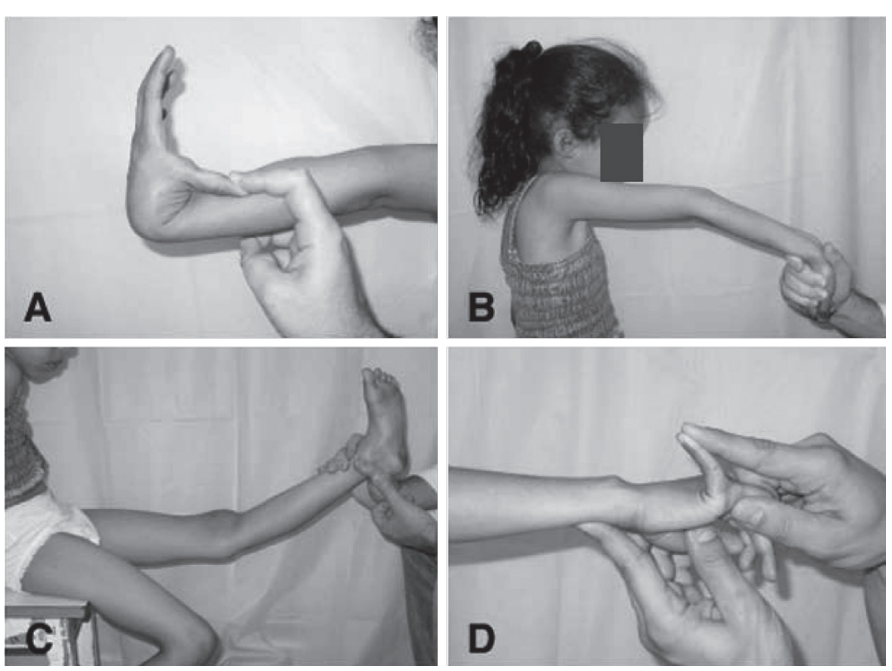

Figura 1 - A) Aposição do polegar com a face ventral do antebraço; B) Hiperextensão passiva do cotovelo; C) Hiperextensão do joelho; D) Extensão passiva do quinto dedo.

1. Extensão passiva dos quirodáctilos mínimos além de $90^{\circ}$.

2. Aposição passiva de cada polegar à face flexora de seu respectivo antebraço.

3. Hiperextensão passiva dos cotovelos, além de $10^{\circ}$.

4. Hiperextensão dos joelhos, além de $10^{\circ}$.

5. Flexão do tronco, partindo da posição ereta, com os joelhos totalmente estendidos, de modo que as palmas das mãos repousem no solo.

A positividade para a frouxidão ligamentar é considerada quando os indivíduos totalizam cinco ou mais pontos.

Quanto à avaliação da presença ou não de PP, todas as crianças foram examinadas mediante a utilização de aparelho de podoscopia com luz e classificados conforme a largura do istmo em relação ao antepé na imagem plantar $^{(13)}$, sendo assim graduados:

Normal - A largura do istmo corresponde a menos da metade da largura total do antepé;

Grau 1 -A largura do istmo supera a metade da largura do antepé, mas não ultrapassa 2/3 dessa mesma largura; 
Grau 2 - A largura do istmo supera 2/3 da largura do antepé, mas não a ultrapassa;

Grau 3 - A largura do istmo é superior à largura do antepé;

Grau 4 - Surgimento do arco lateral.

Para facilidade da interpretação dos resultados obtidos, consideramos somente dois grupos, ou seja, o normal e o portador de PP.

A análise estatística dos resultados foi realizada por um profissional especializado na área de estatística médica que levou em consideração a natureza das distribuições e as variáveis estudadas. Em todos os testes fixou-se em 0,05 ou 5\% o nível de rejeição da hipótese de nulidade e assinalamos com asterisco (*) os valores significantes.

\section{RESULTADOS}

Com o objetivo de avaliar a correlação entre faixa etária e a presença de frouxidão ligamentar, os integrantes foram divididos em dois grupos: zero a sete anos e maior que sete anos. Do total de crianças examinadas, $147(44,8 \%)$ apresentavam frouxidão ligamentar enquanto $181(55,2 \%)$ não apresentavam. De acordo com os dados apresentados na Tabela 1, observamos que houve diferença estatisticamente significante entre a presença ou não de frouxidão nas duas faixas etárias $(\mathrm{p}<0,001 *)$, sendo maior nas crianças com idade abaixo dos sete anos. Esta associação também foi estatisticamente significante quando avaliamos o sexo feminino $(\mathrm{p}<0,001 *)$ e o sexo masculino isoladamente $\left(\mathrm{p}<0,001^{*}\right)$.

Tabela 1 - Distribuição de frequência de indivíduos, separados por faixa etária, de acordo com a presença ou não de frouxidão ligamentar.

\begin{tabular}{|c|c|c|c|c|}
\hline & Faixa etária & Frequência & $\%$ & Total \\
\hline \multirow{2}{*}{ Com frouxidão } & $<7$ anos & 26 & 17,69 & 147 \\
\hline & $>7$ anos & 121 & 82,31 & \\
\hline \multirow{2}{*}{ Sem frouxidão } & $<7$ anos & 5 & 2,76 & 181 \\
\hline & $>7$ anos & 176 & 96,24 & \\
\hline Total & & & & 328 \\
\hline
\end{tabular}

Quanto à correlação entre o sexo e a frouxidão ligamentar, encontramos predomínio da hipermobilidade nos indivíduos do sexo feminino correspondendo a $51,72 \%$ do total. Esta associação foi estatisticamente significante, pelo teste do Qui-quadrado $\left(p=0,025^{*}\right)$. Da mesma maneira, o grupo de indivíduos do sexo feminino apresentou um percentual maior de frouxidão do que o masculino.
Quanto à distribuição das crianças que apresentam ou não PP, encontramos ausência deste em 197 (60,1\%), enquanto $131(39,9 \%)$ apresentam PP. Quando esta variável foi correlacionada com a presença ou não da frouxidão ligamentar, notamos uma associação estatística significante $\left(\mathrm{p}=0,003^{*}\right)$, uma vez que o grupo com PP apresenta um percentual maior $(54,96 \%)$ de frouxidão que o grupo com pé normal $(38,07 \%)$, conforme demonstra a Tabela 2. Esta associação também foi observada quando analisamos o sexo masculino individualmente $(\mathrm{p}=0,001 *)$. Entretanto, o mesmo não foi verificado para o sexo feminino $(p=0,300)$.

Tabela 2 - Distribuição de frequência de indivíduos de acordo com a presença ou não de pé-plano e frouxidão ligamentar.

\begin{tabular}{c|c|c|c|c}
\hline & Pé & Frequência & $\%$ & Total \\
\hline \multirow{2}{*}{ Com frouxidão } & Normal & 75 & 51,02 & 147 \\
\cline { 2 - 5 } & Plano & 72 & 48,98 & \\
\hline \multirow{2}{*}{ Sem frouxidão } & Normal & 122 & 67,4 & 181 \\
\cline { 2 - 5 } & Plano & 59 & 32,6 & \\
\hline Total & & \multicolumn{4}{|c}{ Probabilidade $=0,003^{*}$}
\end{tabular}

Observamos que não houve associação entre a frouxidão e o grupo étnico $(\mathrm{p}=0,934)$ e entre a frouxidão e o lado dominante $(\mathrm{p}=0,831)$ quando aplicado o teste do Qui-quadrado.

Verificamos também que não houve associação entre a presença de frouxidão e o lado dominante, considerandose isoladamente o sexo feminino $(p=0,496)$ e o masculino $(\mathrm{p}=0,901)$, quando aplicado o teste exato de Fischer.

A Figura 2 e a Tabela 3 mostram a distribuição percentual dos indivíduos que apresentam pé plano e frouxidão ligamentar, de acordo com as faixas etárias (considerando intervalo de um ano para cada subgrupo) e o sexo masculino e o feminino.

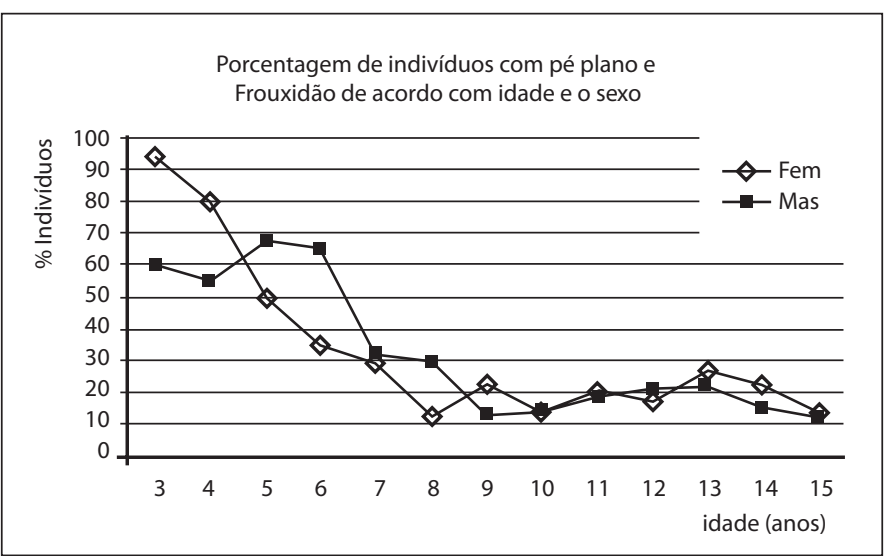

Figura 2 - Porcentagem de Indivíduos com pé plano e frouxidão de acordo. com a idade e o sexo. 
Tabela 3 - Porcentagem de associação entre pé plano e frouxidão ligamentar de acordo com a idade e o sexo.

\begin{tabular}{l|c|c|c|c|c|c|c|c|c|c|c}
\hline Idade (anos) & 3 & 4 & 5 & 6 & 7 & 8 & 9 & 10 & 11 & 12 & 13 \\
\hline Feminino (\%) & 95 & 80 & 50 & 35 & 29 & 12 & 23 & 14 & 21 & 17 & 27 \\
\hline Masculino(\%) & 60 & 55 & 67 & 65 & 32 & 29 & 13 & 14 & 19 & 21 & 22 \\
\hline
\end{tabular}

\section{DISCUSSÃO}

A normalidade, de acordo com nosso vernáculo, compreende um espectro de situações que estão relacionadas a algo natural ou habitual. Entretanto, a dificuldade em estabelecer parâmetros confiáveis de normalidade pode muitas vezes induzir o médico a tratamentos desnecessários quando consideramos alguns aspectos clínicos na infância. A história natural de muitos eventos que participam do desenvolvimento da criança vem sendo frequentemente estudada, gerando uma série de novas evidências que permitem o médico decidir pela melhor conduta para cada caso. Particularmente, no caso do pé plano flexível, percebemos que a indicação de tratamentos específicos como órteses, calçados especiais e palmilhas têm sido abandonados ao longo dos anos pelo reconhecimento de incontestes pesquisas ${ }^{(14)}$ embora sejam ainda muitas vezes aplicados na infância ${ }^{(4,15)}$.

A amplitude do movimento das articulações diminui conforme a progressão da idade. Este decréscimo é mais acentuado na idade escolar e mais lento ao longo da vida adulta $^{(16,17)}$. Este fato também está diretamente relacionado com os diferentes grupos étnicos ${ }^{(16)}$. Além disso, verificamos que a hipermobilidade é maior nos indivíduos do sexo feminino ${ }^{(16-22)}$ e no lado não dominante ${ }^{(16,17)}$.

A hipermobilidade articular pode ser tanto um achado isolado como também pode estar agregada a uma variedade de síndromes genéticas ${ }^{(16,23)}$, observando-se uma prevalência de, aproximadamente, $17 \% \%^{(19)}$ (variando de $4,9 \%$ a $29,8 \%$ ).

Desde 1967 é reconhecida uma síndrome da hipermobilidade que é caracterizada pela frouxidão ligamentar associada com uma sintomatologia específica das articulações $\operatorname{acometidas}^{(17,19,24,25)}$. Acredita-se que os indivíduos com esta característica sejam suscetíveis aos problemas articulares como doenças degenerativas, luxações e sinovites ${ }^{(16,19,24)}$. Quando existe uma queixa específica (5\% a 7\% nas crianças na idade escolar e $4 \%$ a $5 \%$ nos adultos $)^{(19)}$, verificamos com maior frequência o acometimento do joelho, seguido da coluna e do punho ${ }^{(21)}$. Nestas circunstâncias, podem eventualmente ser reconhecidas afecções patológicas, como síndrome de Ehlers-Danlos, síndrome de Marfan, osteogênese imperfeita ${ }^{(19,20)}$, pseudoxantoma elástico, hiperlisinemia, homocistinúria ${ }^{(19)}$ e síndrome de Down ${ }^{(9)}$.
A análise global da flexibilidade é baseada na avaliação de determinados movimentos articulares que refletem a condição geral do indivíduo. Existem diversos métodos de exame descritos na literatura para graduar a frouxidão ligamentar, sendo que cada autor defende sua validade e aplicabilidade $e^{(16,18,25-27)}$. Entretanto, além de confiável, um bom método deve ser facilmente reprodutível ${ }^{(16)}$.

A metodologia proposta por Beighton e Horan é uma das mais utilizadas na literatura medica ${ }^{(16,17)}$ como teste de rastreamento preliminar devido à sua elevada sensibilidade $^{(25)}$. Neste teste, são considerados separadamente os lados direito e esquerdo, embora encontremos autores que não refutam ser necessária esta distinção, pois defendem que os dimídios corporais são simétricos $^{(16,17,25)}$. Em nossa pesquisa, a correlação desta variável clínica é analisada de acordo com o lado dominante, não verificando a associação estatística pelo teste de Fischer.

Após a determinação da presença ou não da frouxidão ligamentar, dividimos nosso material em dois grupos distintos de acordo com faixas etárias (menor que sete anos e maior que sete anos), de forma a estudar a associação entre estas variáveis. Observamos que a hipermobilidade é mais frequente nas crianças com idade abaixo dos sete anos $(\mathrm{p}<0,001 *)$. Da mesma maneira, notamos que o grupo de indivíduos do sexo feminino apresenta um percentual maior de frouxidão do que o masculino. Este dado pode ser apreciado na Figura 2, na qual percebemos nítido decréscimo dos índices de frouxidão de acordo com a progressão da idade. Não concordamos com os trabalhos que agrupam os indivíduos sem distinção quanto ao sexo, desconsiderando esta variável.

Em relação à etiologia do PP flexível, acreditamos na direta relação com a hipermobilidade articular e, portanto, com a frouxidão ${ }^{(3)}$. Contudo, encontramos somente um estudo relevante com características semelhantes na literatura $^{(4)}$ correlacionando a frouxidão ligamentar e o PP. Por definição, o PP caracteriza-se pelo colapso do arco longitudinal, considerado normal em repouso, no momento da descarga de peso do indivíduo ${ }^{(4)}$. Alguns autores consideram que o diagnóstico desta condição 
deva ser feito pela exclusão de outras causas patológicas de $\mathrm{PP}^{(14)}$. Entretanto, concordamos com a literatura de que estes pés raramente são sintomáticos. $\mathrm{O}$ tratamento, quando indicado, está restrito aos pacientes com queixas dolorosas importantes ${ }^{(28)}$. Ressaltamos que a decisão sobre a melhor proposta terapêutica deve estar sempre relacionada com o conhecimento preciso da história natural desta afecção.

Quanto à frequência do PP na amostra estudada, observamos sua presença em 39,9\% dos casos. O grupo com PP apresentou um percentual maior de frouxidão do que o grupo com o pé normal $\left(\mathrm{p}=0,003^{*}\right)$. Quando avaliamos isoladamente os sexos, observamos uma associação entre a presença de frouxidão e faixa etária, pois o grupo com idade maior que sete anos apresentou um percentual menor de crianças com frouxidão do que o grupo com idade menor que sete anos.

Analisando a Figura 2, podemos observar que a frequência de PP e frouxidão difere de acordo com os sexos e sofre declinação que se estabiliza ao redor dos oito anos persistindo em, aproximadamente, $20 \%$ dos casos. Este comportamento se assemelha aos relatados em estudos de outros autores, embora estas variáveis tenham sido avaliadas apenas de forma isolada.

A avaliação clínica dos pés de populações normais, a fim de estabelecer parâmetros de normalidade, tem sido objeto comum de estudo ${ }^{(29-31)}$. Os dados destes trabalhos demonstram que a frequência de PP diminui conforme a progressão da idade. Nossos achados corroboram a opinião destes autores, assim como também percebemos que há decréscimo da frouxidão ligamentar de modo proporcionalmente direto.

Entretanto, em nossa amostra, observamos diferença estatisticamente significante entre os sexos considerandose a evolução do PP, o que difere do resultado de outros pesquisadores $^{(31-33)}$.

O exame clínico para a determinação se o pé é ou não plano é bastante difícil e controverso, devido ao amplo espectro da normalidade ${ }^{(34)}$. Entretanto, quando a sua manifestação é extrema, o seu diagnóstico torna-se naturalmente óbvio ${ }^{(34)}$. Nenhum teste clínico é provido de total confiabilidade, sendo que inúmeros métodos de avaliação são propostos na abordagem do PP. A simples extensão passiva do hálux (teste de Jack) parece ser uma das manobras mais confiáveis, quando avaliada em conjunto com o grau de valgismo do retropé. O exame da impressão plantar, obtida pelo podograma ou podoscopia, também é considerado um método fidedigno de avaliação ${ }^{(14)}$.
Diversos autores expõem suas metodologias de avaliação para qualificar o $\mathrm{PP}^{(14)}$. Preferimos a classificação em cinco tipos exposta anteriormente ${ }^{(13)}$ por ser de fácil reprodução e interpretação, obtida pela visão plantar do pé com o auxílio de podoscópio com luz. Apesar de muitos preferirem a utilização da impressão plantar ${ }^{(29-31,35)}$, a aplicação exata deste método requer cuidados. $\mathrm{O}$ exame de crianças muito pequenas torna-se às vezes complexo pela pouca cooperação e inquietude dos indivíduos durante as avaliações. Qualquer movimentação do pé sobre o papel para a obtenção do podograma irá modificar o desenho real do apoio analisado.

Acreditamos que a realização de trabalhos que envolvam uma grande quantidade de indivíduos é bastante complexa quando analisamos uma determinada variável clínica $^{(21)}$, pois cada exame demanda bastante tempo e acurácia na avaliação, cuja dificuldade foi percebida durante a execução desta pesquisa.

\section{CONCLUSÃO}

A avaliação da hipermobilidade articular é comum na prática diária do ortopedista, e a sua prevalência é variável na população mundial. Pode estar relacionada com condições patológicas do aparelho locomotor como a instabilidade multidirecional do ombro, luxação recorrente da patela, PP hipermóvel com provável alteração do tecido colágeno. Como método de avaliação, preferimos a metodologia de Beighton e Horan por ser de fácil reprodução e ter alto índice de confiança quando aplicada.

Após a realização de análise estatística encontramos:

1. A frouxidão ligamentar é mais frequente na idade menor que sete anos;

2. O grupo de crianças do sexo feminino apresenta percentual maior de frouxidão quando comparado com o do masculino;

3. O grupo de crianças portadoras de PP apresenta percentual maior de frouxidão quando comparado com o grupo com pé normal;

4. Considerando-se separadamente o sexo masculino e o feminino, observa-se percentual maior de PP e frouxidão quando a idade é menor que sete anos;

5. Considerando-se isoladamente o sexo masculino, o grupo com PP apresenta um percentual maior de frouxidão que o grupo com pé normal; e

6. Não há associação entre a presença de frouxidão e cor, assim como frouxidão e lado dominante. 


\section{REFERÊNCIAS}

1. Cavanagh PR, Rodgers MM. The arch index: a useful measure from footprints. J Biomech. 1987;20(5):547-51.

2. Stewart SF. Human gait and the human foot: an ethnological study of flatfoot. II. Clin Orthop Relat Res. 1970;70:124-32.

3. Ferciot CF. The etiology of developmental flatfoot. Clin Orthop Relat Res. 1972;85:7-10.

4. Rose GK, Welton EA, Marshall T. The diagnosis of flat foot in the child. J Bone Joint Surg Br. 1985;67(1):71-8.

5. Silman AJ, Haskard D, Day S. Distribution of joint mobility in a normal population: results of the use of fixed torque measuring devices. Ann Rheum Dis. 1986;45(1):27-30.

6. Beighton P, Solomon L, Soskolne CL. Articular mobility in an African population. Ann Rheum Dis. 1973;32(5):413-8.

7. Qamra SR, Deodhar SD, Jit I. Podographical and metrical study for pes planus in a northwestern Indian population. Hum Biol. 1980;52(3):435-45.

8. Ilfeld, FW. Pes planus - military significance and treatment with simple arch support. JAMA. 1944:124:281-3.

9. Beighton P, Horan F. Orthopaedic aspects of the Ehlers-Danlos syndrome. J Bone Joint Surg Br. 1969;51(3):444-53.

10. Tachdjian MO. Congenital convex pes valgus. In: Pediatric orthopedics. 2nd ed. Philadelphia: WB Saunders;, 1990. p. 2557-69,

11. Fairbank JC, Pynsent PB, Phillips H. Quantitative measurements of joint mobility in adolescents. Ann Rheum Dis. 1984 Apr;43(2):288-94.

12. Rodriguez N, Volpe RG. Clinical diagnosis and assessment of the pediatric pes planovalgus deformity. Clin Podiatr Med Surg. 2010;27(1):43-58.

13. Valenti V. Ortesis del pie. Madrid: Medicina Panamericana Editorial; 1979. 175p.

14. Hatt WS, Davis LA. Analysis of the foot in infant, radiographic criteria and clinical aspects. South Med J. 1954;50:720.

15. Staheli LT, Chew DE, Corbett M. The longitudinal arch. A survey of eight hundred and eighty-two feet in normal children and adults. J Bone Joint Surg Am. 1987;69(3):426-8.

16. Backx FJ, Erich WB, Kemper AB, Verbeek AL. Sports injuries in school-aged children. An epidemiologic study. Am J Sports Med. 1989;17(2):234-40.

17. Barry RJ, Scranton PE Jr. Flat feet in children. Clin Orthop Relat Res. 1983;(181):68-75.

18. Decoster LC, Vailas JC, Lindsay RH, Williams GR. Prevalence and features of joint hypermobility among adolescent athletes. Arch Pediatr Adolesc Med. 1997;151(10):989-92.
19. Krivickas LS, Feinberg JH. Lower extremity injuries in college athletes: relation between ligamentous laxity and lower extremity muscle tightness. Arch Phys Med Rehabil. 1996;77(11):1139-43.

20. Chung PK, Yuen CK. Criterion-related validity of sit-and-reach tests in university men in Hong Kong. Percept Mot Skills. 1999;88(1):304-16.

21. Didia BC, Dapper DV, Boboye SB. Joint hypermobility syndrome among undergraduate students. East Afr Med J. 2002;79(2):80-1.

22. Larsson LG, Baum J, Mudholkar GS. Hypermobility: features and differential incidence between the sexes. Arthritis Rheum. 1987;30(12):1426-30.

23. Seow CC, Chow PK, Khong KS. A study of joint mobility in a normal population. Ann Acad Med Singapore. 1999;28(2):231-6.

24. Rikken-Bultman DG, Wellink L, van Dongen PW. Hypermobility in two Dutch school populations. Eur J Obstet Gynecol Reprod Biol. 1997;73(2):189-92.

25. Brodie DA, Bird HA, Wright V. Joint laxity in selected athletic populations. Med Sci Sports Exerc. 1982;14(3):190-3.

26. Araújo CG. Correlação entre diferentes métodos lineares e adimensionais de avaliação da mobilidade articular. Rev Bras Cien Mov. 2000;8(1):25-32.

27. Bulbena A, Duró JC, Porta M, Faus S, Vallescar R, Martín-Santos R. Clinical assessment of hypermobility of joints: assembling criteria. J Rheumatol. 1992;19(1):115-22

28. Volpon JB. Footprint analysis during the growth period. J Pediatr Orthop. 1994;14(1):83-5.

29. Ilfeld FW. Pes Planus: a method of mensuration. Radiology. 1945;44:355-6.

30. Larsson LG, Baum J, Mudholkar GS, Srivastava DK. Hypermobility: prevalence and features in a Swedish population. Br J Rheumatol. 1993;32(2):116-9.

31. Bordelon RL. Hypermobile flatfoot in children. Comprehension, evaluation, and treatment. Clin Orthop Relat Res. 1983;(181):7-14.

32. Chang JH, Wang SH, Kuo CL, Shen HC, Hong YW, Lin LC. Prevalence of flexible flatfoot in Taiwanese school-aged children in relation to obesity, gender, and age. Eur J Pediatr. 2010;169(4):447-52.

33. Chen JP, Chung MJ, Wang MJ. Flatfoot prevalence and foot dimensions of 5 - to 13-year-old children in Taiwan. Foot Ankle Int. 2009;30(4):326-32.

34. Chandler FA. Children's feet, normal and presenting common abnormalities. Am J Dis Child. 1942;63:1136.

35. Villarroya MA, Esquivel JM, Tomás C, Moreno LA, Buenafé A, Bueno G. Assessment of the medial longitudinal arch in children and adolescents with obesity: footprints and radiographic study. Eur J Pediatr. 2009;168(5):559-67. 